\title{
Pseudomyxoma extraperitonei of ovarian origin mimicking a psoas abscess
}

\author{
I. Chamisa
}

Received: 11 November 2009 / Accepted: 24 December 2009/Published online: 27 January 2010

(C) Springer-Verlag 2010

\begin{abstract}
Pseudomyxoma peritonei (PMP) is a rare condition consisting of mucinous ascites, most commonly arising from mucinous tumours of the appendix and occasionally from the ovary. Very rarely, mucinous deposits arise in the retroperitoneum without intraperitoneal involvement. This has been termed pseudomyxoma extraperitonei (PME). Our 48-year-old female patient presented with PME mimicking a psoas abscess for which retroperitoneal exploration and biopsy was done with accidental breach of the peritoneum. She declined further surgery and re-presented 4 years later with disseminated PMP. She underwent debulking surgery including right oophorectomy which was confirmed as the primary tumour source.
\end{abstract}

Keywords Pseudomyxoma peritonei $\cdot$ Psoas abscess · Pseudomyxoma extraperitonei $\cdot$ Retroperitoneal mucinous adenocarcinoma

\section{Introduction}

Pseudomyxoma peritonei (PMP) first described by Werth is a rare and poorly understood disease characterised by abundant extracellular mucin in the peritoneum [1]. Clinical presentation of patients with PMP includes features of acute appendicitis, increasing abdominal girth, inguinal or umbilical hernia. The entity of pseudomyxoma extraperitonei (PME) in which mucinous implants arise in

\footnotetext{
I. Chamisa $(\square)$

Kalafong Hospital Department of General Surgery,

University of Pretoria,

Private Bag X 396,

Pretoria, South Africa

e-mail: charms@doctors.org.uk
}

the retroperitoneum without intraperitoneal involvement is even more uncommon. Our literature search revealed only 12 cases of PME that have been reported in the English literature and in almost all the cases, mucinous tumours of the appendix was described as the origin [2]. We report a rare case of PME arising from the ovary and mimicking a psoas abscess.

\section{Case presentation}

A 48-year-old female patient presented to the gynaecology clinic with a 2-month history of right iliac fossa pain, constipation and increased abdominal girth. General examination revealed a well-looking patient with a low-grade fever and normal pulse rate. Full blood count showed microcytic hypochromic anaemia with a normal white cell count and C-reactive protein was marginally raised at $26.2 \mathrm{mg} / \mathrm{l}$. Abdominal examination confirmed a firm mass in the right iliac fossa extending to the right flank. Computed tomography (CT) showed a solid-cystic, multiloculated retroperitoneal mass encasing the right psoas muscle (Fig. 1). She was then referred to the surgical outpatient clinic for further assessment. With a presumptive diagnosis of a psoas abscess, she underwent exploration which revealed a multi-cystic mass with gelatinous material and histology confirmed PMP. The peritoneum was accidentally breached during the procedure. The patient was then offered further debulking surgery and she declined. She was however given a follow-up date in the surgical outpatient clinic but never turned up. Poor followup compliance of patients is common in our setting. She then re-presented 4 years later with increased abdominal girth associated with a mass in the right flank and the typical 'jelly belly' appearance of PMP (Fig. 2). Carci- 


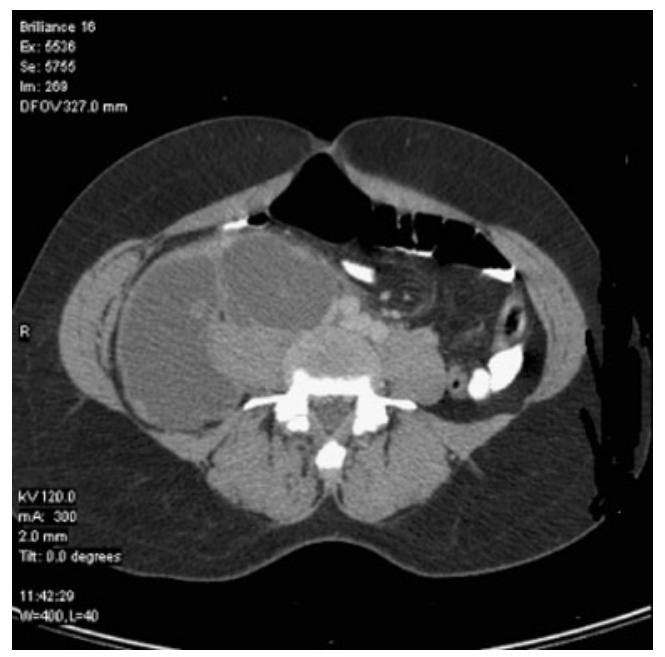

Fig. 1 CT scan on initial presentation showing a large solid-cystic, multiloculated retroperitoneal mass encasing the right psoas muscle and confined to the retroperitoneum (pseudomyxoma extraperitonei)

noembryogenic antigen (CEA) and CA 19.9 were both elevated at $6 \mathrm{ng} / \mathrm{ml}$ and $75 \mathrm{U} / \mathrm{mg}$ respectively. CT scan (Fig. 3) confirmed disseminated intra-peritoneal and extraperitoneal PMP with spread through the posterior abdominal wall into the subcutaneous tissue. She underwent de-bulking surgery including right oophorectomy for a huge ovarian mass followed by a course of intravenous infusion cisplatin and cyclophosphamide. We do not have facilities for intraperitoneal chemotherapy. Histology confirmed the right ovary as the primary tumour origin and there was no identifiable tumour in the appendix.

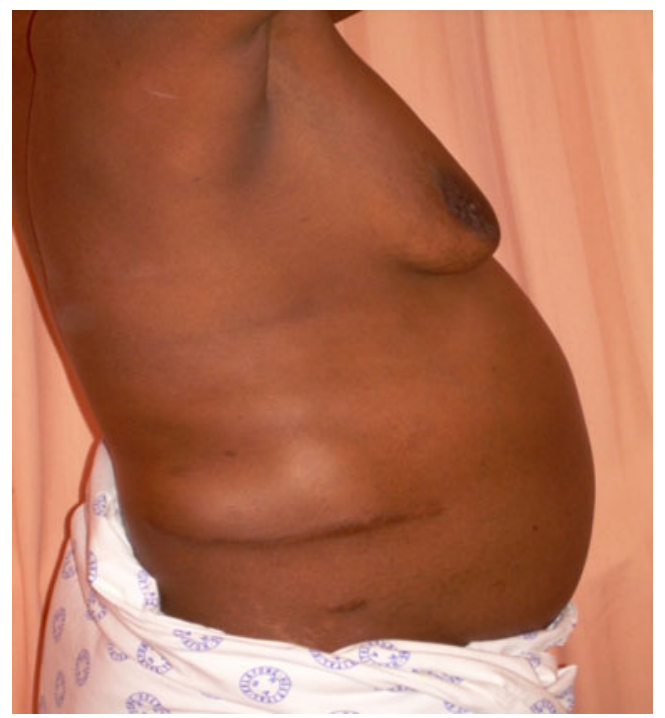

Fig. 2 Patient picture showing the typical 'jelly belly' abdomen of PMP and a right flank mass with an overlying scar from a previous retroperitoneal exploration for a presumed diagnosis of psoas abscess 4 years previously

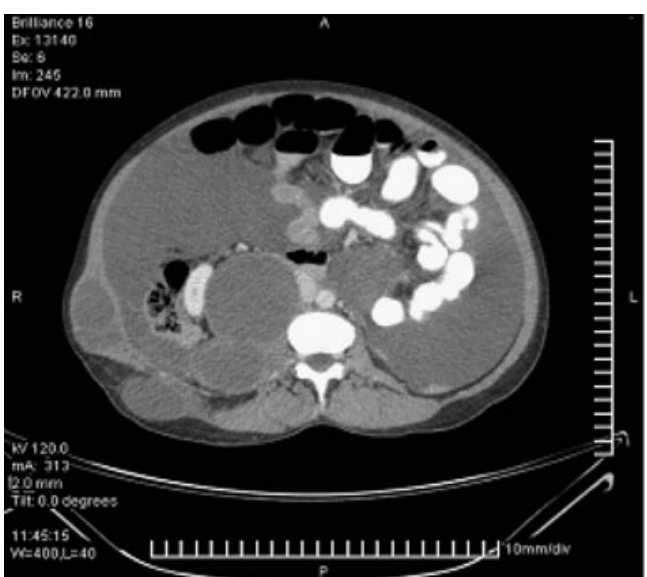

Fig. 3 CT scan showing disseminated intraperitoneal and extraperitoneal pseudomyxoma peritonei with a mass in the right iliac fossa extending subcutaneously to the right flank and displacement of the aorta and inferior vena cava to the left

Follow-up involved a comprehensive physical examination, CT scan of abdomen and pelvis 3 months after surgery and 6 months later including tumour markers CEA and carbohydrate antigen (CA) 19.9. She remains well 18 months after debulking surgery.

\section{Discussion}

PMP is the clinical term for the debilitating syndrome of grossly apparent gelatinous ascites associated with peritoneal deposits of mucinous tumours. Most of this syndrome is mainly attributed to spread from the appendix and rarely from mucinous ovarian neoplasms. Mucocele of the appendix is a rare condition found in $0.2-0.3 \%$ of appendicectomies and describes an abnormal mucous deposition in the lumen of the appendix [2]. PMP involving only the retroperitoneum was first described by Bonann in 1948 [3]. This was later termed as 'pseudomyxoma extraperitonei' by Moran [4] and later as 'pseudomyxoma retroperitonei' by Shelton et al. [5].

Our patient presented with clinical and radiological features mimicking a psoas abscess. Previous literature reports have described PMP presenting as an iliacus abscess, scrotal mass or hydronephrotic kidney [6-8]. Other abdominal cystic lesions, including cystic degeneration of giant myoma, have also been reported to mimic mucinous adenocarcinoma and PMP [9]. PME is a very rare condition in which the retroperitoneal space is filled with thick, gelatinous material with sparing of the intraperitoneum. In the described literature cases, the origin was diagnosed as or suspected to be rupture of appendiceal mucinous tumour into the retroperitoneum in almost all cases. Other potential primary sites include mucinous neoplasm of the ovary, the 
uterus, bowel, urachus, common bile duct, and pancreas. In our patient, the primary tumour origin was confirmed as ovarian and this has rarely been reported.

CT scan findings are pathognomonic for PMP and demonstrate the characteristic distribution of mucinous ascites, which can be differentiated from normal ascites by unusual density properties. In early stage disease, omentum, sigmoid, ovaries and sub-hepatic and ileocaecal regions may be involved, with visceral sparing. In late stage diseases, generally all regions are affected and abundant mucinous ascites causes compression of small bowel and impression of the liver. This was the case in our patient who had hydronephrosis and displacement of the aorta and inferior vena cava. When CT confirms PMP, there is a role for serum tumour markers, CEA and CA 19.9 in the completion of the diagnostic work-up as these tumour markers are raised in most patients as demonstrated in the index case.

The mainstay of treatment of PMP remains aggressive surgical debridement of all gelatinous deposits. Sugarbaker reported curative treatment of malignant PMP with aggressive cyto-reductive surgery and adjuvant intraperitoneal chemotherapy [10]. Surgical de-bulking is the standard treatment, but combined modality treatment, consisting of aggressive peritonectomy with intra-operative hyperthemic intra-peritoneal chemotherapy is the new recommended standard approach [11].

After combined modality treatment, patients should be monitored for recurrence or progressive disease. CT scan is recommended for detecting progressive disease at least 3 months after treatment as a basis for further disease surveillance. Other useful tests in detecting disease in the post-surgical period are the tumour markers CEA and CA 19.9 which also act as prognostic factors for survival. Mortality from PMP is usually secondary to intestinal obstruction, fistula formation, peritonitis, or pulmonary embolus. Our patient remains well 18 months after de-bulking surgery and an intravenous infusion course of cisplatin and cyclophosphamide.

\section{Conclusion}

PMP is a rare disease with a poor prognosis. Early recognition of the clinical syndrome is the initial step to improve the prognosis, and CT imaging is the recommended choice of radiological assistance in the diagnostic process. The clinical and radiological features of PME can mimic a psoas abscess and we therefore recommend that all atypical presentations of psoas abscess or retroperitoneal masses should undergo full diagnostic work-up including tumour markers (CEA, CA 19.9) to exclude PME before exploration. Furthermore, in PME or undiagnosed retroperitoneal masses, it is imperative that an intact and complete removal of the mass be achieved without spillage of the contents in the peritoneum to prevent intraperitoneal extension of the disease. This case also serves to emphasise the importance of involving both gynaecologists and surgeons in the investigations and management of atypical retroperitoneal masses in females. In addition to debulking surgery, we further emphasise the need to explore the appendix and ovaries during primary surgery for retroperitoneal cystic lesions in order to assist in the determination of the primary source. Finally, although the majority of PMP and PME arise from the appendix, ovarian origin should be considered as illustrated in our patient.

Conflict of interest There is no actual or potential conflict of interest in relation to this article.

\section{References}

1. Werth R (1884) Pseudomyxoma peritonei. Arch Gynaecol 24:100-118

2. Koizumi H, Noguchi H (1999) Pseudomyxoma retroperitonei with spontaneous skin fistula. Abdom Imaging 24:193-195

3. Bonnan LJ, Davis JG (1948) Retroperitoneal mucocele of the appendix. A case report with characteristic roentgen features. Radiology 51:375-382

4. Moran CG, Morgan RH (1988) Pseudomyxoma extraperitonei. J R Soc Med 81:668-669

5. Shelton MW, Morian JP, Radford DM (1994) Pseudomyxoma retroperitonei associated with appendiceal cystadenoma. Am Surg 60:958-960

6. Edrees WK, Hannon RJ (1999) Pseudomyxoma extra-peritonei: a rare presentation mimicking an iliacus abscess. The Ulster Med J 68:38-39

7. Baker WC, Goldman LB, de Vere White RW (1988) Pseudomyxoma peritonei presenting as a scrotal mass. J Urol 139(4):821-822

8. Mor Y, Leibovitch I, Garnick A, Avigad I, Jonas P, Goldwasser B (1996) Pseudomyxoma extraperitonei: an unusual presentation mimicking a huge hydronephrotic kidney. Clin Radiol 51:221-222

9. Kirshtein B, Sebbag G, Pak I (2005) Giant cystic lesion mimicking Pseudomyxoma peritonei. Gynaecol Surg 2(1):39-41

10. Sugarbaker PH, Landy D, Jaffe G, Pascal R (1990) Histologic changes induced by intraperitoneal chemotherapy with 5-fluorouracil and mitomycin $\mathrm{C}$ in patients with peritoneal carcinomatosis from cystadenocarcinoma of the colon or appendix. Cancer 65:1496-1501

11. Smeenk RM, Verwaal VJ, Zoetmulder FAN (2007) Pseudomyxoma peritonei. Cancer Treat Rev 33:138-145 